\section{Application of Method of Small Parameters to Multi-Niche Population Genetic Models*}

Samuel Karlin ${ }^{\dagger}$ and James McGregor

Stanford University

Received October 4, 1971

\section{INTRODUCTION}

In theoretical population genetics it has been customary to analyze models in which most or all of the parameters are assigned specific values. For example, one of the earliest models discussed by Haldane was concerned with the rate of elimination of a recessive lethal, and in this model the viabilities assigned to genotypes $A A, A a, a a$ are $1,1,0$, respectively. Implicit in the study of such models is the assumption that the conclusions derived, in their general qualitative aspects, and to some extent in their quantitative features, will be applicable to more "realistic" models in which the parameter values are only approximately the same as those assigned in the model. In the above example an assignment of $b_{1}, b_{2}, b_{3}$ as the respective viabilities, with $b_{1}$ and $b_{2}$ nearly 1 and $b_{3}$ nearly zero, should not alter the qualitative features of the model and should result in only small changes of the important quantitative aspects. A gene perfectly dominant in all its pleiotropic effects coupled with a recessive allele perfectly lethal when homozygous, even in the complex natural environment, comprise a structure of little interest, except to the extent that it may serve as an idealized approximation for cases in which the dominance and the lethality are not quite perfect.

This paper is one of several companion papers in which a theory of perturbation of parameters in deterministic genetic systems is developed. The most naive application of this perturbation theory would be to show that the qualitative and quantitative conclusions derived from models, such as the aforementioned model of Haldane, do indeed persist under small perturbations of the parameters in the model. However, the theory has more valuable and less obvious applications in the analysis of complex genetic population systems composed of a number of interacting subpopulations, or generally subsystems. A number of examples are presented below which illustrate this kind of application.

* Work was supported in part by National Institute of Health Grant USPRS 10452-09. + Weizmann Institute of Science, Israel and Stanford University.
A general perspective on the general principles underlying the "method of small parameters" and ramifications of them and their implications are set forth in Karlin and McGregor [1972]. For applications of this theory to the study of multilocus selection balance, see [1973].

The examples of this paper deal with populations existing in two or more niches (colonies, subpopulations), subject to natural or sexual selection in each niche, and with interaction between the niches mediated by migration. If the gene flow induced by migration is weak then the system can be regarded as a perturbation of the simpler system in which there is no migration and the niches are completely isolated. Here we regard the migration rates between the niches as the parameters subject to perturbation. Features of such a model which occur for certain special values of the parameters, and which are of significance to the population biologist, must be subject to only small quantitative variations when the parameters are slightly changed. In the examples below, the significant feature studied is the existence of a stable polymorphism.

To make the discussion more precise suppose, to start with, that the population has two alleles $A, a$ at a locus and occupies two niches. Let $x_{1}$ and $x_{2}$ be the A-gene frequencies in niche 1 and niche 2, respectively. When the niches are completely isolated, the gene frequencies in the next generation $x_{1}{ }^{\prime}$ and $x_{2}{ }^{\prime}$ will be given by certain transformation laws

$$
\begin{aligned}
& x_{1}^{\prime}=f_{1}\left(x_{1}\right) \\
& x_{2}^{\prime}=f_{2}\left(x_{2}\right)
\end{aligned}
$$

which take account of the mating system, mutation and selection pressures, etc., which operates in the niches. When there is migration between the niches the transformation of gene frequencies will be of the form

$$
\begin{aligned}
& x_{1}^{\prime}=g_{1}\left(x_{1}, x_{2}\right)=\left(1-m_{1}\right) f_{1}\left(x_{1}\right)+m_{1} f_{2}\left(x_{2}\right) \\
& x_{2}^{\prime}=g_{2}\left(x_{1}, x_{2}\right)=m_{2} f_{1}\left(x_{1}\right)+\left(1-m_{2}\right) f_{2}\left(x_{2}\right)
\end{aligned}
$$

where $m_{1}, m_{2}$ are migration rates. When $m_{1}, m_{2}$ are small (1.2) is nearly the same as (1.1).

A pair of gene frequencies $\left(z_{1}, z_{2}\right)$ is an equilibrium point for (1.2) if $g_{i}\left(z_{1}, z_{2}\right)=z_{i}, i=1,2$. The equilibrium point is called locally stable if, for every initial frequency vector $\left(x_{1}, x_{2}\right)$ near $\left(z_{1}, z_{2}\right)$, the sequence of vectors

$$
\begin{gathered}
\left(x_{1}^{\prime}, x_{2}^{\prime}\right),=x^{\prime}=g\left(x_{1}, x_{2}\right) \\
\left(x_{1}^{\prime \prime}, x_{2}^{\prime \prime}\right),=x^{\prime \prime}=g\left(\begin{array}{ll}
x_{1}^{\prime} & x_{2}^{\prime}
\end{array}\right)
\end{gathered}
$$

obtained from $\left(x_{1}, x_{2}\right)$ by iteration of (1.2), converges to $\left(z_{1}, z_{2}\right)$. (For a more 
general and more precise definition of locally stable, see Karlin and McGregor (1972).

We prove in that paper that if (1.1) has a locally stable equilibrium point $\left(y_{1}, y_{2}\right)$ then every neighborhood $U$ of $\left(y_{1}, y_{2}\right)$ contains a neighborhood $V$ of $\left(y_{1}, y_{2}\right)$ such that for all sufficiently small $m_{1}, m_{2}$ the transformation (1.2) maps $V$ into itself. Thus for every initial vector $\left(x_{1}, x_{2}\right)$ in $V$ the successive iterates (1.3) all lie in $V$. Under additional hypotheses, concerning derivatives of $f_{1}$ and $f_{2}$, we show in [1972] that $V$ contains a unique locally stable equilibrium point for (1.2). In the examples below, which have been chosen for their analytic simplicity, this latter statement is directly verifiable.

Thus the existence of a locally stable equilibrium point is a qualitative feature preserved under small perturbations.

It should be noted that an unstable equilibrium point need not survive unde small perturbations. For example, the function $f(x)=x / 2+2 x^{2}(1-x)$ has $x=1 / 2$ as an unstable equilibrium point but for small positive $\epsilon, f(x)-\epsilon x$ has no equilibrium point $x$ with $0<x<1$. The existence of a locally stable equilibrium point in a model is biologically significant, indeed it may serve to explain a state of fixation or of polymorphism in a population which the model is designed to imitate. For this reason the existence of a locally stable equilibrium point should be preserved under perturbation of the parameters. In contrast, an unstable equilibrium point has no such significance, it represents a state unlikely to be observed.

Particularly interesting applications arise from (1.2) when the locally stable equilibrium $\left(y_{1}, y_{2}\right)$ of $(1.1)$ is $(1,0)$ or $(0,1)$. That is, in the absence of migration, niche 1 tends to fix on one allele and in niche 2 on the other allele. The conclusion then is that when the migration flow is small the system still has a locally stable equilibrium in which the population in niche 1 is comprised mostly of one allele, that in niche 2 mostly of the other allele.

For convenience of reference we now state two general principles concerning perturbations of parameters in genetic systems. These principles are proved as theorems and discussed in considerable detail in the companion paper of this issue. The transformation law for the gene frequencies in a deterministic genetic model has the form

$$
x_{i}^{\prime}=f_{i}\left(x_{1}, x_{2}, \ldots, x_{k} ; m_{1}, \ldots, m_{p}\right), \quad i=1, \ldots, k
$$

where $m_{1}, \ldots, m_{p}$ are parameters which may be migration rates, selection coefficients, recombination fractions, virulence coefficients, and so on. The possible frequency vectors $x=\left(x_{1}, \ldots, x_{k}\right)$ for system (1.4) comprise a polyhedron $\Omega$ in $k$-dimensional Euclidean space, and the parameters form a vector $m=\left(m_{1}, \ldots, m_{p}\right)$ lying in a set $M$ in $p$-space. In a shorthand notation (1.4) can be written as

$$
x^{\prime}=f(x ; m) \text {. }
$$

We assume that for each $m$ the system (1.4a) determines a continuous map of $\Omega$ into itself, and that the functions $f_{i}(x ; m)$ are jointly continuous in the $k+p$ variables $x_{1}, \ldots, x_{k}, m_{1}, \ldots, m_{p}$. The first principle is concerned with the preservation of locally stable fixed points.

PRINCIPLE I. Suppose that for a certain parameter vector $\tilde{m}$ the system (1.4a) has a locally stable fixed point $y \in \Omega$. Then, given any neighborhood $U$ of $y$, there is a neighborhood $V$ of $y, V \subseteq U$, such that for every $m$ sufficiently close to $\tilde{m}$, (1.4a) maps $V$ into itself.

The small neighborhood $V$ is then a region of stability or region of no escape for the slightly perturbed system, and from a practical viewpoint has the same significance as a locally stable fixed point.

The mapping (1.4) or (1.4a) is called continuously differentiable if the partial derivatives

$$
\left(\partial f_{i} / \partial x_{j}\right), \quad i, j=1, \ldots, k
$$

are jointly continuous functions of the $k+p$ variables $x_{1}, \ldots, x_{k}, m_{1}, \ldots, m_{p}$. The stability nature of an equilibrium point $y$ can often be settled by determining the eigenvalues of the gradient matrix $f^{\prime}(y ; m)$, that is, the $k \times k$ matrix whose $i, j$ element is $\partial f_{i} / \partial x_{j}$ evaluated at the point $y$. Specifically, if all the eigenvalues $\lambda$ satisfy $|\lambda|<1$ then $y$ is locally stable, but if $y$ is an interior point of $\Omega$ and at least one eigenvalue $\lambda$ satisfies $|\lambda|>1$ then $y$ is unstable. The second principle, $*$ (see [1972] for its proof) is concerned with the variation in the number of fixed points when the parameters are perturbed.

PrInciple II. Assume that the mapping (1.4a) of $\Omega$ into itself is continuously differentiable. Suppose for a parameter value $\tilde{m}$ the mapping has exactly $\nu$ fixed points $y^{(1)}, \ldots, y^{(v)}$ in $\Omega$ and at each of these fixed points all eigenvalues $\lambda$ of the gradient matrix satisfy $\lambda \neq 1$. Then there exist small neighborhoods $U^{(1)}, \ldots, U^{(v)}$ of $y^{(1)}, \ldots, y^{(v)}$ respectively such that the mapping (1.4a) has, for all $m$ sufficiently close to $\tilde{m}$ at most $\nu$ fixed points in $\Omega$ and at most one fixed point in each $U^{(\alpha)}$. Further, if $y^{(\alpha)}$ is locally stable there actually exists a locally stable fixed point $z^{(\alpha)}$ in the corresponding $U^{(\alpha)}$ for the transformation (1.4a).

\section{Models with Selection Balance in Two Niches}

The structure of multiniche selection models has been partially investigated by many authors [e.g., Levene (1953), Moran (1959), Prout (1968), Parsons (1963), and Eyland (1971)]. Consider the case of two alleles $A$ and $a$ at a locus

* This is stated as Principle III in the companion paper of this issue. 
in a population divided into two niches (subpopulations, colonies, etc.). We will assume random mating occurs in each niche, selection occurs in each niche during maturation, and the adults migrate between the niches just prior to the next round of random mating. Let $x_{1}$ and $x_{2}$ be the A-gene frequencies in niches 1 and 2, respectively after random mating. Let the relative selective values of the genotypes $A A, A a, a a$ be $b_{1}, b_{2}, b_{3}$ respectively in niche 1 and $c_{1}, c_{2}, c_{3}$ respectively in niche 2 . Suppose the sizes of niches 1 and 2 are $N_{1}$ and $N_{2}$ respectively, and that after maturation a proportion $m_{1}\left(m_{2}\right)$ of individuals migrate from niche 1 (niche 2 ) to the other niche. Then the gene frequencies after the next round of random mating will be

$$
\begin{aligned}
x_{1}^{\prime}= & \frac{\left(1-m_{1}\right) N_{1}}{\left(1-m_{1}\right) N_{1}+m_{2} N_{2}} \frac{b_{1} x_{1}{ }^{2}+b_{2} x_{1}\left(1-x_{1}\right)}{b_{1} x_{1}{ }^{2}+2 b_{2} x_{1}\left(1-x_{1}\right)+b_{3}\left(1-x_{1}\right)^{2}} \\
& +\frac{m_{2} N_{2}}{\left(1-m_{1}\right) N_{1}+m_{2} N_{2}} \frac{c_{1} x_{2}{ }^{2}+c_{2} x_{2}\left(1-x_{2}\right)}{c_{1} x_{1}^{2}+2 c_{2} x_{2}\left(1-x_{2}\right)+c_{3}\left(1-x_{2}\right)^{2}} \\
x_{2}^{\prime}= & \frac{m_{1} N_{1}}{m_{1} N_{1}+\left(1-m_{2}\right) N_{2}} \frac{b_{1} x_{1}^{2}+b_{2} x_{1}\left(1-x_{1}\right)}{b_{1} x_{1}^{2}+2 b_{2} x_{1}\left(1-x_{1}\right)+b_{3}\left(1-x_{1}\right)^{2}} \\
& +\frac{\left(1-m_{2}\right) N_{2}}{m_{1} N_{1}+\left(1-m_{2}\right) N_{2}} \frac{c_{1} x_{2}^{2}+c_{2} x_{2}\left(1-x_{2}\right)}{c_{1} x_{1}^{2}+2 c_{2} x_{2}\left(1-x_{2}\right)+c_{3}\left(1-x_{2}\right)^{2}}
\end{aligned}
$$

These equations are of the form

where

$$
\begin{aligned}
& x_{1}{ }^{\prime}=\left(1-m_{21}\right) f_{1}\left(x_{1}\right)+m_{21} f_{2}\left(x_{2}\right) \\
& x_{2}{ }^{\prime}=m_{12} f_{1}\left(x_{1}\right)+\left(1-m_{12}\right) f_{2}\left(x_{2}\right)
\end{aligned}
$$

$$
\begin{aligned}
m_{21} & =\frac{m_{2} N_{2} / N_{1}}{1-m_{1}+m_{2} N_{2} / N_{1}} \\
m_{12} & =\frac{m_{1} N_{1} / N_{2}}{1-m_{2}+m_{1} N_{1} / N_{2}} \\
f_{1}\left(x_{1}\right) & =\frac{b_{1} x_{1}^{2}+b_{2} x_{1}\left(1-x_{1}\right)}{b_{1} x_{1}^{2}+2 b_{2} x_{1}\left(1-x_{1}\right)+b_{8}\left(1-x_{1}\right)^{2}} \\
f_{2}\left(x_{2}\right) & =\frac{c_{1} x_{2}^{2}+c_{2} x_{2}\left(1-x_{2}\right)}{c_{1} x_{2}{ }^{2}+2 c_{2} x_{2}\left(1-x_{2}\right)+c_{3}\left(1-x_{2}\right)^{2}}
\end{aligned}
$$

For given $N_{1}$ and $N_{2}$ the exchange coefficients $m_{21}, m_{12}$ will be small when $m_{1}$ and $m_{2}$ are small.

Special cases of the above model have been investigated by several authors. Moran (1962, Chap: 11) examined the case $b_{1}>b_{2}>b_{3}$ and $c_{1}<c_{2}<c_{3}$ with all these parameters close to 1 and $N_{1}=N_{2}, m_{1}=m_{2}$. Maynard Smith (1966) treated a variation of this same model with the $A$ gene dominant. Notice that $A$ is advantageous in niche 1 while $a$ is favored in niche 2 . By approximate procedures (specifically replacing (2.2) by a set of difference equations) Moran established the existence of a stable polymorphism for the special choices $b_{1}=c_{3}$ and $b_{3}=c_{1}$ such that selection operates in a symmetric fashion in each niche. With the same methodology, Eyland (1971) has extended the analysis to some other cases. Moran (1962, p. 175) feels on plausibility grounds that so long as selection operates in opposite directions in the two subpopulations, a stable polymorphism is possible. This intuition is not universally correct. For moderate or large migration rates the two subpopulations become sufficiently mixed that the ultimate fate of the population is determined by the combined effects of the selection pressures.

To illustrate the possibilities when the exchange coefficients are large although selection operates in different directions in the two niches, we analyze the case

$$
\begin{array}{ccc}
b_{1}=b_{2}=1, \quad b_{3}=1-s, & 0<s<1, \\
c_{1}=c_{2}=1, \quad c_{3}=1+\sigma, & \sigma>0, \\
m_{12}=m_{21}=1 / 2 &
\end{array}
$$

Here the $A$ gene is dominant and is advantageous in niche 1 but disadvantageous in niche 2 . The values $m_{12}=m_{21}=1 / 2$ correspond to thorough mixing of the two populations at the time of mating. Indeed we have $x_{1}{ }^{\prime}=x_{2}{ }^{\prime}$ so the A-gene frequency has the same value $x$ in both niches and obeys the transformation law

$$
x^{\prime}=g(x)=\frac{1}{2} f_{1}(x)+\frac{1}{2} f_{2}(x)=\frac{1}{2}\left[\frac{x}{1-s(1-x)^{2}}+\frac{x}{1+\sigma(1-x)^{2}}\right]
$$

All solutions of $g(x)=x$ are readily found. In addition to $x=0$ (fixation on $a$ ) and $x=1$ (fixation on $A$ ) there may be a solution which satisfies $x(1-x)=$ $(\sigma-s) / 2 \sigma s$. We obtain the following cases.

(i) $s<\frac{1}{2}, \sigma<s$. There is no polymorphic equilibrium $(0<x<1)$, $g^{\prime}(0)>1, g^{\prime}(1)=1, g^{\prime \prime}(1)>0$ and eventual fixation on $A$ occurs for any initial frequency $x_{0}>0$.

(ii) $s<\frac{1}{2}, s<\sigma<s /(1-2 s)$. There is a unique internal equilibrium $0<x<1$, which is stable, and the gene frequency converges to this value if the initial frequency $x_{0}$ is not 0 or 1 .

(iii) $\left.s<\frac{1}{2}, \sigma\right\rangle s /(1-2 s)$. There is no internal equilibrium, $x=0$ is stable and eventual fixation on $a$ occurs for any initial frequency $x_{0}<1$.

If $s>\frac{1}{2}$ then $x=0$ is always unstable.

(iv) $s>\frac{1}{2}, \sigma<s$. There is eventual fixation on $A$ for any initial frequency $x_{0}>0$.

(v) $s>\frac{1}{2}, \sigma>s$. There is a unique internal equilibrium which is approached from any initial frequency $x_{0}$ not 0 or 1 . 
These examples show that merely having each gene advantageous in one niche, and permitting migration, does not ensure a polymorphism. The ultimate outcome is sensitive to the orders of magnitude of all the parameters. Similar results apply whenever $m_{21}$ and $m_{12}$ are near $1 / 2$. On the other hand, we show below that when selection operates in different directions in the two niches then a polymorphism will always be possible provided the intermigration rates are sufficiently small, concordant with Principle I of Section 1.

\section{Multi Niche Models with DisRuptive Selection}

I. We next carry out a complete analysis of the system (2.1) for the special case (disruptive selection), $b_{1}=b_{3}=c_{1}=c_{3}=1, b_{2}=c_{2}=1-s, 0<s<1$, $N_{1}=N_{2}, m_{1}=m_{2}=m$.

\begin{tabular}{cccccc}
$A A$ & Niche I & \multicolumn{3}{c}{ Niche II } \\
1 & $A a$ & $a a$ & $A A$ & $A a$ & $a a$ \\
$1-s$ & 1 & 1 & $1-s$ & 1
\end{tabular}

The equations (2.1) can now be cast in the form

where

$$
\begin{aligned}
& x_{1}{ }^{\prime}-\frac{1}{2}=(1-m) f\left(x_{1}\right)+m f\left(x_{2}\right) \\
& x_{2}{ }^{\prime}-\frac{1}{2}=m f\left(x_{1}\right)+(1-m) f\left(x_{2}\right)
\end{aligned}
$$

$$
f(x)=\frac{x-1 / 2}{1-2 \sin (1-x)}
$$

The corresponding single niche system has precisely three equilibria with the equilibrium values $x=1, \frac{1}{2}$ or 0 , and $x=1,0$ are locally stable while $x=(1 / 2)$ is unstable. Consequently, when $m=0$, the two niche system has exactly $3^{2}=9$ equilibria of which $2^{2}=4$ are locally stable. For these latter 4 the equilibrium frequency vectors $\left(x_{1}, x_{2}\right)$ are $(1,1),(0,0),(1,0),(0,1)$. According to the general principles of Section 1, the coupled system (3.1) will, for sufficiently small $m$, have at most 9 (in this case the bound is achieved) equilibrium points in the square $0 \leqslant x_{1}, x_{2} \leqslant 1$, of which exactly 4 will be locally stable. Two of these locally stable ones will have frequency vectors near $(1,0)$ and $(0,1)$ and thus will correspond to stable polymorphisms. (The other two locally stable ones are the fixation states $(1,1)$ and $(0,0)$ exactly.) In order to maintain the stable polymorphisms near $(1,0)$ and $(0,1)$ it will be necessary that $m$ is not too large. However it is clear that, to some extent, an increase of the migration rate $\boldsymbol{m}$ could be compensated for by increasing the selective disadvantage $s$ of the heterozygotes.
To determine the equilibria with $x_{1}-\frac{1}{2}$ and $x_{2}-\frac{1}{2}$ not both zero, we eliminate these variables from the equilibrium version of (3.1), obtaining

$u=m v /(2 s v-m) \quad$ where $\quad u=x_{1}\left(1-x_{1}\right), \quad v=x_{2}\left(1-x_{2}\right)$.

Evidently, $u, v$ are subject to the constraints $0 \leqslant u, v \leqslant(1 / 4)$. From (3.2) and the equilibrium version of (3.1) we arrive at the single equation for $v$,

$m^{2}[m-2(s-2 m) v](1-2 s v)^{2}=(1-4 v)(m-2 s v)[m-(1-m) 2 s v]^{2}$. (3.3)

The pure state equilibria $(1,1)$ and $(0,0)$ correspond to the solution $u=v=0$. Another solution with $u=v$ is found, namely $u=v=m / s$ which is valid when, and only when, $0<m / s<(1 / 4)$. The other two roots of (3.3) are obtained by solving the quadratic equation

$$
8 s^{2}(1-m)^{2} v^{2}+(s+2 m-2 s m)(m-2 s v)=0 .
$$

A little analysis reveals that this quadratic has two roots, satisfying $0<v<(1 / 4)$ if

$$
s>6 m-4 m^{2}
$$

and no such roots if the reverse inequality holds. Let $v_{1}, v_{2}$ be the roots of the quadratic when (3.5) is valid. If $v=x_{2}\left(1-x_{2}\right)$ is one of these roots then the other root is $m v /(2 s v-m)=u=x_{1}\left(1-x_{1}\right)$.

The equilibrium frequencies $x_{1}, x_{2}$ are determined from the solutions $v$ of (3.3), using (3.2) and taking account of the fact, evident from (3.1), that if $u, v$ are not zero then $x_{1}-\frac{1}{2}$ and $x_{2}-\frac{1}{2}$ must be of opposite sign. The solution $v=m / s$ yields two equilibria

$$
P^{ \pm}: x_{1}=\frac{1}{2} \pm \frac{1}{2} \sqrt{1-(4 m / s)}, x_{2}=\frac{1}{2} \mp \frac{1}{2} \sqrt{1-(4 m / s)}
$$

and the solutions $v_{1}, v_{2}$ yield four more equilibria

$$
\begin{aligned}
& Q^{ \pm}: x_{1}=\frac{1}{2} \pm \frac{1}{2} \sqrt{1-4 v_{1}}, x_{2}=\frac{1}{2} \mp \frac{1}{2} \sqrt{1-4 v_{2}}, \\
& R^{ \pm}: x_{1}=\frac{1}{2} \pm \frac{1}{2} \sqrt{1-4 v_{2}}, x_{2}=\frac{1}{2} \mp \frac{1}{2} \sqrt{1-4 v_{1}} .
\end{aligned}
$$

A standard local linear analysis establishes the nature of the stability of these equilibria. The results are summarized in the following table and the analysis is outline below.

TABLE I

\begin{tabular}{ccc}
\hline Equilibrium & Conditions for existence & Conditions for local stability \\
\hline$(0,0)$ & always & always \\
$(1,1)$ & always & always \\
$\left(\frac{1}{2}, \frac{1}{2}\right)$ & always & never \\
$P^{ \pm}$ & $s>4 m>0$ & $s>6 m-4 m^{2}$ \\
$Q^{ \pm}, R^{ \pm}$ & $s>6 m-4 m^{2}$ & never \\
\hline
\end{tabular}


The following features of the results are noted as typical and similar to features of other models.

(a) For sufficiently small $m$ there are 9 equilibria of which 4 are locally stable. The other 5 equilibria of course lie on curves which separate the domains of attraction of the four locally stable equilibria, as in Fig. 1.

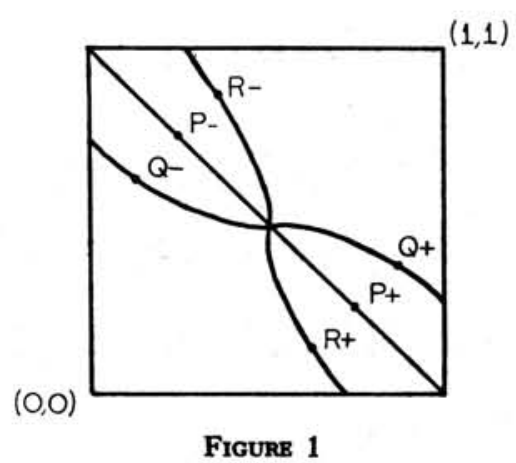

(b) The symmetric type equilibria $P_{+}$and $P_{-}$exist if $m<m^{*}=s / 4$ but are locally stable only if the more restrictive condition (3.5) holds, or equivalently

$$
m<m^{* *}=\frac{s}{3+\sqrt{9-4 s}} .
$$

The critical value $m^{* *}$ for local stability is smaller than the critical value $m^{*}$ for existence.

(c) As $m$ increases the number of interior equilibria and their stability classification varies in a complicated way, reflecting the complex interaction of - the migration and selection pressures.

The analysis of local stability for the symmetric equilibria of (3.1) can be done most expeditiously in a general setting. Consider the transformation

$$
\begin{aligned}
& x^{\prime}=(1-m) f(x)+m f(y) \\
& y^{\prime}=m f(x)+(1-m) f(y)
\end{aligned}
$$

where $|f(x)| \leqslant 1$ and suppose

$$
f(x)=1-f(1-x) \quad \text { for all } x .
$$

This assumption is satisfied in example (3.1).
We concentrate on an equilibrium $\left(x_{0}, y_{0}\right)$ of the form $y_{0}=1-x_{0}$, $0<x_{0}<1$. The local linear analysis of (3.6) at $\left(x_{0}, x_{0}\right)=\left(x_{0}, 1-x_{0}\right)$ leads to the linear system

$$
\begin{aligned}
& X^{\prime}=(1-m) f^{\prime}\left(x_{0}\right) X+m f^{\prime}\left(1-x_{0}\right) Y \\
& Y^{\prime}=m f^{\prime}\left(x_{0}\right) X+(1-m) f^{\prime}\left(1-x_{0}\right) Y .
\end{aligned}
$$

Note that the antisymmetry property (3.7) implies $f^{\prime}\left(x_{0}\right)=f^{\prime}\left(1-x_{0}\right)$. The eigenvalues of the system (3.9) are readily found to be

$$
\lambda_{1}=f^{\prime}\left(x_{0}\right), \quad \lambda_{2}=f^{\prime}\left(x_{0}\right)(1-2 m)
$$

and therefore the equilibrium under consideration is locally stable if and only if $\left|f^{\prime}\left(x_{0}\right)\right|<1$.

For the system (3.1) there are three symmetric equilibria, $P_{+}, P_{-}$and $\left(\frac{1}{2}, \frac{1}{2}\right)$, the first two existing only if $0<4 m<s$. For $P_{+}$and $P_{-}$the condition of local stability is $(1-s+2 m) /(1-2 m)^{2}<1$ or equivalently $s>6 m-4 m^{2}$ as in (3.5). An easy computation shows that $f^{\prime}(1 / 2)=1 /(1-s / 2)$ so $(1 / 2,1 / 2)$ is not locally stable.

II. The more general version of (2.1) when

$$
\begin{gathered}
b_{1}=b_{3}=c_{1}=c_{3}=1, \\
b_{2}=1-s, \quad c_{2}=1-t, \quad(-\infty<s, t<1), \\
N_{1}=N_{2}, \quad m_{1}=m_{2}=m
\end{gathered}
$$

can be investigated by similar methods. We describe briefly the results. Note we are allowing cases of both heterozygote advantage and disadvantage (since $-\infty<s, t<1$ ) independently in each niche. They are summarized below. (Throughout we assume $m \leqslant 1 / 2$.)

(i) The pure equilibria $(0,0)$ and $(1,1)$ are locally stable if and only if

$$
s+t>\max (-[2 m /(1-m)],-[s t(1-2 m) / m]) \text {. }
$$

(Remember that $s$ and $t$ may be negative and range independently from $-\infty$ to 1 .) The characteristic polynomial for the equilibria $(0,0)$ and $(1,1)$ is

$$
f(\lambda)=\lambda^{2}-\lambda(1-m)(2-s-t)+(1-s)(1-t)(1-2 m)
$$

and the inequality (3.9) decides when both roots are in magnitude less than 1.

(ii) The symmetrical equilibrium $\left(\frac{1}{2}, \frac{1}{2}\right)$ is locally stable if and only if

$$
\min [(s t / 2 m),(4 m+s t) /(1+m)]>s+t .
$$


It can be proved that the pure equilibria and the symmetric equilibrium can never be stable together.

Aside from $((1 / 2),(1 / 2))$ the possible equilibria are determined as the roots of the equation in $v=x_{2}\left(1-x_{2}\right)$,

$m^{2}[m s-2 t v(s-2 m)](1-2 t v)^{2}-(1-4 v) s(m-2 t v)[m-(1-m) 2 t v]^{2}=0$.

Any admissible root must satisfy $0 \leqslant v \leqslant(1 / 4)$. Equation (3.11) has $v=0$ as a root corresponding to the two pure equilibria $(0,0)$ and $(1,1)$. It is an easy matter to establish that it has either one or three additional admissible roots, yielding two or six internal equilibria.

It is perhaps worth underscoring some facts that emerge from the analysis of the preceding models of this section.

(i) It is a familiar proposition that disruptive selection for a single population leads to fixation. It is tempting to propose the same conclusion for the multi niche version of this model. However for a multi (at least two) niche situation with disruptive selection in force, say, in each niche, then provided migration flow is sufficiently slight, stable polymorphisms are possible. The precise bound of the migration coefficient $m$ assuring polymorphism in model I of Section 3 is determined by the inequality

$$
s>6 m-4 m^{2} \text {. }
$$

Subject to this condition, there exists 4 stable equilibria ( 2 polymorphic and 2 pure) and 5 unstable equilibria.

(ii) There are genetic models (e.g., the first model above) where exactly 3 internal equilibria exist none of which are stable. Indeed this is the case provided

$$
4 m<s<6 m-4 m^{2}
$$

This proliferation of unstable equilibria is not a common occurrence in the analysis of genetic models.

(iii) Results analogous to those of Section 3 obtain for general multiniche multiallelic selection models. Moreover, approximate locations of all the stable polymorphic equilibria of the system are easily forthcoming provided all migration rates among the niches are small. This is most easily accomplished by appealing to the general principle of Section 1 .

(iv) It is important to note the existence of other cases of stable polymorphism in the presence of large migration rates among the niches. For these cases the task of discerning complete criteria is analytically prohibitive.
III. Multi-Niche Multiallele Migration Selection. Consider a diploid population, with $n$ alleles $A_{1}, A_{2}, \ldots, A_{n}$ at a locus, undergoing random mating and subject to disruptive selection. Let $x_{i}$ be the $A_{i}$-gene frequency, so the frequencies of the genotypes $A_{i} A_{i}$ and $A_{i} A_{j}$ after reproduction are $x_{i}{ }^{2}$ and $2 x_{i} x_{j}$ respectively $(i \neq j)$. Let $w_{i j}$ be the (relative) fitness of $A_{i} A_{j}$, where $w_{i j}=w_{j i}$. The mean fitness of the population is

$$
W(x)=\sum_{i} \sum_{j} w_{i j} x_{i} x_{j}
$$

and the gene frequencies in the next generation will then be

$$
x_{i}^{\prime}=x_{i} \sum_{j} w_{i j} x_{j} / W(x)=f_{i}\left(x_{1}, \ldots, x_{n}\right) .
$$

It is well known that an equilibrium frequency vector $x=\left(x_{1}, \ldots, x_{n}\right)$ of (3.13) is locally stable if and only if $x$ is an isolated local maximum of the fitness function $W(x)$.

We assume the selection is disruptive in the sense that for each $i, j$

$$
w_{i i}>w_{i j} \quad \text { if } j \neq i \text {. }
$$

Under this circumstance the only locally stable equilibria are the pure ones, those with one $x_{i}=1$, all other $x_{i}$ zero. To see this, suppose $x=\left(x_{1}, x_{2}, \ldots, x_{n}\right)$ is an equilibrium frequency vector with, say, both $x_{1}$ and $x_{2}$ not zero. From (3.13)

$$
\sum_{j} w_{i j} x_{j}=W(x)
$$

for each $i$ with $x_{i} \neq 0$. We compute $W(x)$ where $\tilde{x}_{1}=x_{1}+h, \tilde{x}_{2}=x-h$, $\tilde{x}_{i}=x_{i}$ for $i>2$. The result is, using (3.15),

$$
W(\tilde{x})=W(x)+h^{2}\left(w_{11}-w_{12}-w_{21}+w_{22}\right) .
$$

Here the coefficient of $h^{2}$ is positive because of (3.14), so $W(\tilde{x})>W(x)$ for small $h \neq 0$. It follows that $W$ does not have a local maximum at $x$, so $x$ is not locally stable.

Now suppose we have $k$ replicas of the above population in $k$ niches with small migration between niches. For simplicity we assume the niches are of the same size, with the same fitness coefficients, and that after reproduction a small proportion $\boldsymbol{m}$ of the population of each niche migrate to each of the other niches. Let $x_{\alpha i}$ be the $A_{i}$-gene frequency in niche $\alpha$. The transformations for this system are

$$
\begin{array}{r}
x_{\alpha i}^{\prime}=[1-(k-1) m] f_{i}\left(x_{\alpha 1}, \ldots, x_{\alpha n}\right)+m \sum_{\beta \neq \alpha} f_{i}\left(x_{\beta 1}, \ldots, x_{\beta n}\right) \\
i=1, \ldots, n, \quad \alpha=1, \ldots, k .
\end{array}
$$


When $m=0$ the locally stable equilibria of the system (3.17) correspond to pure populations in each of the $k$ niches, the allele maintained in each niche being arbitrary. It follows that in this case for all sufficiently small $m>0$ the $k$ niche system has locally stable equilibria in which $k$ alleles are present in every niche. In particular, if $k \geqslant n$ then for migration rate $m$ sufficiently small, a polymorphic equilibrium can be maintained involving all types in every niche.

The following converse proposition has interesting implications (consult the discussion).

IV. Assume (3.14) prevails. Let $k<n$ (i.e., the number of niches is fewer than the possible number of alleles.) Then for small positive intermigration flow $m$ there exists no stable $n$ allele polymorphism.

Thus for the case of complete disruptive selection (the selection parameters may differ over successive niches) involving $\boldsymbol{n}$ possible alleles in each niche, the number of available niches must equal or exceed $\boldsymbol{n}$ in order to assure a polymorphic balance. The validation of this assertion invokes the conclusions of both Principles I and II. We present the detailed arguments in the case of $n=3, k=2$ which embraces the essential ideas for the general case.

Under the conditions of (3.14) we know that in each separate niche the vertex equilibria are locally stable while the edge equilibria and interior equilibrium are all unstable. Moreover, except for special relations among the $w_{i j}$ none of the eigenvalues of the gradient matrix at any equilibrium equals 1 . An application of Principle II yields the existence of a single stable equilibrium in the neighborhood of each combination of the vertex points

$$
(1,0,0),(0,1,0),(0,0,1)
$$

with an arbitrarily chosen one of these from each niche. All other possibilities of equilibrium arising in the two niche system are unstable points. In the case at hand with $n=3, k=2$, there are $n^{k}=3^{2}=9$ stable equilibrium of which three are the obvious pure states of fixation.

Consider next the equilibrium with allelic frequencies near $(1,0,0)$ and $(0,1,0)$ in niches 1 and 2 , respectively. Such exists by virtue of Principle I. A further application of Principle I to the corresponding two-niche model where allele 3 is missing affirms the fact of the existence of an equilibrium involving only the first two alleles and none of the third allele. This equilibrium is necessarily the unique locally stable one near $(1,0,0) \times(0,1,0)$ (by Principle II).

Paraphrasing the above analysis for all pairwise combinations of (3.18), we find that the 6 nonpure stable equilibria each involve exactly 2 alleles. The proof of assertion III in the special case $n=3, k=2$ is complete.

The general formulation of the preceeding result is as follows. For any multiniche ( $k$ niches), multi-allelic ( $n$ alleles) selection model, a stable polymorphism is possible if and only if there exists a stable equilibrium in each separate niche involving say $n_{1}, n_{2}, \ldots, n_{k}$ alleles, respectively, with the property that the total set of alleles occurring among these alleles includes all types.

\section{Polymorphism for a Two-Locus Two-Niche Haploid Population}

Consider a two-locus two-niche population comprised of 4 gamete types with possible alleles $a, A$ and $b, B$ at the first and second locus respectively.

The parameters of the haploid model are listed in the tabulation below:

\begin{tabular}{|c|c|c|c|c|}
\hline Gamete & $A B$ & $A b$ & $a B$ & $a b$ \\
\hline \multirow[t]{2}{*}{$\begin{array}{c}\text { Fitness } \\
\text { Coefficients }\end{array}$} & $\sigma_{1}$ & $\sigma_{2}$ & $\sigma_{3}$ & 1 \\
\hline & $\sigma_{1}^{\prime}$ & $\sigma_{\mathbf{2}}^{\prime}$ & $\sigma_{3}^{\prime}$ & 1 \\
\hline
\end{tabular}

The recombination fraction is denoted by $r$ which is fixed throughout these considerations.

Feldman's work (1971) suggests that in any single niche haploid two locus population practicing random mating, selection pressures alone cannot maintain a stable polymorphism. Thus to achieve polymorphism some other influence apart from selection pressures should be operating. Feldman, from his analysis, argues against the likelihood of finding random mating in nature. He pointed out that an incompatibility mating pattern as that which occurs with certain fungus populations; e.g. Schizophyllum Commune, with no selection forces present, will lead to stable polymorphism. We will prove as an application of our principle of Section 1, that under certain conditions on the selection parameters, a polymorphism is also possible for the multiniche version of the haploid two locus random mating population. It is necessary first to review the conditions, in the single niche model, for local stability of the pure equilibria (see Bodmer and Parsons [1962]). We shall deal first with the situation

$$
\begin{aligned}
& \sigma_{1}>1>\max \left(\sigma_{2}, \sigma_{3}\right) \\
& \sigma_{1}{ }^{\prime}>1>\max \left(\sigma_{2}^{\prime}, \sigma_{3}{ }^{\prime}\right) .
\end{aligned}
$$

Suppose the inequalities above hold. The equilibrium consisting of only the $A B$ gametes is manifestly locally stable since this gamete is selectively superior to all the other gametes in both niches.

If the condition

$$
\sigma_{1}(1-r)<1
$$


is also satisfied then the equilibrium of a pure population comprised only of $a b$ gametes is also locally stable. The validation of this fact is accomplished by a standard local analysis. The remaining two cases of pure populations (equilibria with all $A b$ or all $a B$ types) are not locally stable.

Consider now the two niche situation where the selection parameters satisfy the conditions of (4.1), (4.2) and

$$
\sigma_{1}(1-r)<1, \quad \sigma_{1}{ }^{\prime}\left(1-r^{\prime}\right)<1 .
$$

Therefore, in each individual niche the pure equilibria of $A B$ or $a b$ gametes are each locally stable. If migration flow between niches is sufficiently small then the general principle of Section 1 tells us that there exist two stable equilibria exhibiting a preponderance of $A B$ gametes and a small percentage of $a b$ gametes in niche 1 and a preponderance of $a b$ gametes and a small percentage of $A B$ gametes in niche 2 or the reverse way. Since $r>0$ the gametes $A b$ and $a B$ are necessarily also represented. Therefore two stable polymorphisms are possible under the selection conditions stated provided that the migration rates between niches are sufficiently small.

The same arguments show that stable polymorphism obtains, for example, when the selection parameters fulfill the following requirements

(i) $\sigma_{1}>\max \left(\sigma_{2}, \sigma_{3}, 1\right)$ and $1>\max \left(\sigma_{1}{ }^{\prime}, \sigma_{2}{ }^{\prime}, \sigma_{3}{ }^{\prime}\right)$;

(ii) $\sigma_{1}>1>\max \left(\sigma_{2}, \sigma_{3}\right),(1-r) \sigma_{1}<1$ and $\sigma_{1}{ }^{\prime}>\max \left(\sigma_{2}{ }^{\prime}, \sigma_{3}{ }^{\prime}, 1\right)$;

(iii) analogous cases where the gametes $A b$ and $a B$ are the most fit.

In the circumstance (i), the $A B$ gamete is most advantageous in the first subpopulation, while in the second population the $a b$ gamete is most advantageous. Here a polymorphic equilibrium is possible with mostly $A B$ gametes manifested in niche 1 and mostly $a b$ gametes present in niche 2 .

The precise character of the polymorphic equilibrium can also be ascertained in the circumstances of (ii) and (iii).

The above analysis emphasizes the general tenet that many selection models can produce stable polymorphism by spatially spreading the selection effects (i.e., to several niches) and keeping the interaction between niches sufficiently weak.

\section{Some Multiniche incompatibility Systems}

Workman (1964) introduced a series of incompatibility models aiming to explain the existence of large numbers of polymorphisms in natural plant populations. Analysis of several of his models was elaborated in Karlin and Feldman (1968) who found that in most cases no stable polymorphism obtains.
However, in the light of the general principle of Section 1, we reexamine two of the cases of some interest.

Model 1. Consider a population of genotypes $A_{1} A_{1}, A_{1} A_{2}$ and $A_{2} A_{2}$ where the similarity matings $A_{1} A_{1} \times A_{1} A_{1}, A_{1} A_{2} \times A_{1} A_{2}$, and $A_{2} A_{2} \times A_{2} A_{2}$ are prohibited; i.e., only unlike genotypes may mate. Thus the population consists of three mating types, determined by two alleles at one locus, and only individuals of distinct types can mate. This type of mating behavior (diploid-diploid incompatibility, see Spieth (1971)) is particularly relevant to certain plant populations. Let $u, v, w\left(u^{\prime}, v^{\prime}, w^{\prime}\right)$ denote the frequencies of $A_{1} A_{1}, A_{1} A_{2}$ and $A_{2} A_{2}$ in two successive generations. We obtain the recursion relations

$$
\begin{aligned}
u^{\prime} & =(u v(1+w) / 2(1-u)(1-v))=f_{1}(u, v, w) \\
v^{\prime} & =(u w / 2)((1+v) /(1-u)(1-v))+(1 / 2)=f_{2}(u, v, w) \\
w^{\prime} & =(w v(1+u) / 2(1-v)(1-v))=f_{3}(u, v, v),
\end{aligned}
$$

(see Karlin and Feldman (1968)). The evolutions of a single population is somewhat similar to that resulting from disruptive selection. More specifically, depending on the initial composition whether $u_{0}<w_{0}$ or $u_{0}>w_{0}$, the population approaches the equilibrium $(0,(1 / 2),(1 / 2))$ or $((1 / 2),(1 / 2), 0)$, respectively at a geometric rate.

Consider now two replica populations each independently evolving in accordance with the recursions (5.1). Suppose some light migration (a proportion $m$ ) occurs between each of the populations. The principle of Section 1 asserts that with $m$ sufficiently small, a locally stable polymorphism may be attained. Actually, for the model at hand it is possible to set forth a more precise quantitative description of the relevant equilibria.

Let $u, v, w$ be the equilibrium frequencies of $A_{1} A_{1}, A_{1} A_{2}, A_{2} A_{2}$ in population 1 and $U, V, W$ the corresponding equilibrium frequencies in population 2 . The equations of the global system at equilibrium are

$$
\begin{aligned}
u & =(1-m) f_{1}(u, v, w)+m f_{1}(U, V, W) \\
v & =(1-m) f_{2}(u, v, w)+m f_{2}(U, V, W) \\
w & =(1-m) f_{3}(u, v, w)+m f_{3}(U, V, W) \\
U & =m f_{1}(u, v, w)+(1-m) f_{1}(U, D, W), \quad \text { etc. } \\
V & =\ldots \ldots \ldots \ldots \\
W & =\ldots \ldots \ldots
\end{aligned}
$$

Symmetry considerations suggest we seek a solution with

$$
u=W, \quad w=U \quad \text { and } \quad v=V .
$$


Inserting these values into (5.2) and assuming $u-v \neq 0$ and executing other manipulations we have the identities

$$
\begin{gathered}
(1+z) \frac{v}{2(1-v)}(1-2 m)=v+z \quad \text { where } z=u w \\
(2 v-1)(v+z)=z(1+v) .
\end{gathered}
$$

Equation (5.5) is obtained by adding the first and third equation in (5.2) and cancelling $u+v$. Equations (5.4) and (5.5) are obtained by subtracting and adding the first and third equations of (5.2) and cancelling $u-w$ in one case, $u+w$ in the other. Eliminating $z$ from (5.4) and (5.5), and assuming $v \neq 0$ (the third equation of (5.2) shows $v \geqslant 1 / 2$ ), we obtain

$$
2(1-m) v^{2}-v(1-2 m)-2 m=0 .
$$

This quadratic equation for $v$ has one negative root and one positive root $\tilde{v}$. If $0<m<1 / 2$ then $1 / 2<\tilde{v}<1$, otherwise $\tilde{v} \geqslant 1$. (As $m$ increases from 0 to $1 / 2, \tilde{v}$ increases steadily from $1 / 2$ to 1 .) With $v=\tilde{v}$ we obtain $u v$ from (5.5),

$$
u w v=z=[(\tilde{v}(2 \tilde{v}-1) /(2-\tilde{v})] .
$$

Since $u, w$ must now be determined from $u+w=1-v$ and

$$
u w=((u+w) / 2)^{2}-((u-w) / 2)^{2}=((1-\tilde{v}) / 2)^{2}-((u-w) / 2)^{2}
$$

an actual equilibrium solution will result if and only if $v=\tilde{v}$ satisfies the inequality

or equivalently

$$
[v(2 v-1) /(2-v)]<((1-v) / 2)^{2}
$$

$$
v^{3}+4 v^{2}+v-2<0
$$

There is a unique value $v^{*}, 1 / 2<v^{*}<1$, such that $v$ satisfies (5.7) if $0<v<v^{*}$ but not if $v>v^{*}$.

Thus no equilibrium is obtained if $\tilde{v}>v^{*}$, but if $\tilde{v}<v^{*}$ we solve the system

$$
\begin{aligned}
u+w & =1-\tilde{v} \\
(u-v)^{2} & =(1-\tilde{v})^{2}-4 \tilde{v}(2 \tilde{v}-1) /(2-\tilde{v})
\end{aligned}
$$

and obtain two polymorphic equilibria $\left(u_{+}, \tilde{v}, w_{+}\right)$and $\left.u_{-}, \tilde{v}, w_{-}\right)$where

$$
u_{ \pm}-w_{ \pm}= \pm\left[(1-\tilde{v})^{2}-4 \tilde{v}(2 \tilde{v}-1) /(2-\tilde{v})\right]^{1 / 2},
$$

and the associated values $U, V, W$ are determined by (5.3).
Because of the monotone dependence of $\tilde{v}$ on $m$, there is a unique value $0<m^{*}<1 / 2$, such that $\tilde{v}<v^{*}$ if and only if $m<m^{*}$.

It follows from the general principle of Section 1 that these equilibria are locally stable provided $m$ is sufficiently small, say $m<m^{* *}$ (we carry out a formal proof of the corresponding assertion for Model 2 below). It can be expected that the critical value $m^{* *}$, for stability, is smaller than the critical value $m^{*}$, for existence, of the polymorphisms.

Model 2. In another of Workman's incompatibility models, there are again two alleles $A_{1}, A_{2}$ at one locus in a diploid population, but now only the mating type $A_{1} A_{2} \times A_{1} A_{2}$ is prohibited. We assume the population is distributed in two niches and let the frequencies of $A_{1} A_{1}, A_{1} A_{2}, A_{2} A_{2}$ be $(u, v, w)$ in niche 1 and $(U, V, W)$ in niche 2 . Suppose that after reproduction a proportion $m$ of the population of each niche migrates to the other niche. The relations for the equilibrium frequencies are of the form (5.2) but now

$$
\begin{aligned}
& f_{1}(u, v, w)=u\left(u+\frac{1}{2} v+\frac{1}{2} \frac{v}{1-v}\right) \\
& f_{2}(u, v, w)=v-\frac{1}{2} v^{2}+u w \\
& f_{3}(u, v, w)=v\left(w+\frac{1}{2} v+\frac{1}{2} \frac{v}{1-v}\right) .
\end{aligned}
$$

In a single isolated niche the population approaches the equilibrium $(1,0,0)$ (all $\left.A_{1} A_{1}\right)$ if initially $u>w$, or approaches the equilibrium $(0,0,1)$ (all $A_{2} A_{2}$ ) if initially $u<v$. The two niche population with no migration therefore has a locally stable equilibrium with $u=W=1, v=w=U=V=0$. It readily follows from the general principle of Section 1 that when there is migration between the two niches; there will be a polymorphic locally stable equilibrium with $u$ and $W$ both near 1 provided the migration is sufficiently small. We will analyze this case more completely.

The symmetry of the model suggests we seek an equilibrium solution with $u=W, v=V, w=U$. Assuming $u-w \neq 0$, the conditions to be satisfied are found in the form

$$
\begin{gathered}
1=(1-2 m)\left[1+\frac{v^{2}}{2(1-v)}\right] \\
2 u w=\frac{1}{2} v^{2} .
\end{gathered}
$$

The quadratic equation (5.9) for $v$ has a unique root $\tilde{v}$ such that $0<\tilde{v}<1$ if $0<m<1 / 2$. When $m$ increases from 0 to $1 / 2, \tilde{v}$ increases steadily from 0 to 1 . In order that a genuine equilibrium solution exist with $v=\tilde{v}$ we must have

$$
\tilde{v}^{2}=4 u w<(u+w)^{2}=(1-\tilde{v})^{2}
$$


or $\tilde{v}<1 / 2$. The solution $\tilde{v}$ of (5.9) satisfies $\tilde{v}<1 / 2$ if and only if

$$
m<m^{*}=1 / 10 \text {. }
$$

Let $\tilde{v}$ be the solution of (5.9) when $m<m^{*}$. Then from (5.10)

$$
(u-w)^{2}=(1-\tilde{v})^{2}-4 u w=1-2 \tilde{v}
$$

and

$$
\left(\tilde{u}_{ \pm}, \tilde{v}, \tilde{w}_{ \pm}\right)=\left(\frac{1-\tilde{v}}{2} \pm \frac{\sqrt{1-2 \tilde{v}}}{2}, \tilde{v}, \frac{1-\tilde{v} \pm \sqrt{1-2 \tilde{v}}}{2}\right)
$$

with $\tilde{V}=\tilde{w}, \tilde{V}=\tilde{v}, \tilde{W}=\tilde{u}$, give explicitly the two symmetric equilibria.

The conditions for local stability of the equilibria (5.12) are discerned as follows:

Write the variables of the transformation $u=\tilde{u}+x, v=\tilde{v}+y, w=\tilde{w}+z$, $U=\tilde{U}+X, V=\tilde{V}+Y, \tilde{W}=W+Z$ and $u^{\prime}=\tilde{u}+x^{\prime}, v^{\prime}=\tilde{v}+y^{\prime}$, etc. for the transformed variables. Then execute a local linear analysis (about the equilibrium). Note that there are four independent variables among $\{x, y, z, X, Y, Z\}$ and suggested by symmetry considerations it is convenient to operate in terms of the variables $x-Z, z-X, x+Z, z+X$. The local linear approximation with simplifications and exploiting symmetries yields the equations

$$
\begin{aligned}
x^{\prime}-Z^{\prime}= & {\left[(1-m)\left(\frac{\partial f_{1}}{\partial x}-\frac{\partial f_{1}}{\partial y}\right)+m \frac{\partial f_{3}}{\partial y}\right](x-Z) } \\
& +\left[-(1-m) \frac{\partial f_{1}}{\partial y}-m\left(\frac{\partial f_{3}}{\partial y}+\frac{\partial f_{3}}{\partial z}\right)\right](z-X) \\
z^{\prime}-X^{\prime}= & {\left[-m\left(\frac{\partial f_{1}}{\partial x}+\frac{\partial f_{1}}{\partial y}\right)-(1-m) \frac{\partial f_{3}}{\partial y}\right](x-Z) } \\
& +\left[(1-m)\left(\frac{\partial f_{3}}{\partial z}-\frac{\partial f_{3}}{\partial y}\right)-m \frac{\partial f_{1}}{\partial y}\right](z-X) \\
x^{\prime}+Z^{\prime}= & {\left[(1-m)\left(\frac{\partial f_{1}}{\partial x}-\frac{\partial f_{1}}{\partial y}\right)-m \frac{\partial f_{1}}{\partial y}\right](x+Z) } \\
& +\left[m\left(\frac{\partial f_{3}}{\partial z}-\frac{\partial f_{3}}{\partial y}\right)-(1-m) \frac{\partial f_{1}}{\partial y}\right](z+X) \\
z^{\prime}+X^{\prime}= & {\left[m\left(\frac{\partial f_{1}}{\partial x}-\frac{\partial f_{1}}{\partial y}\right)-(1-m) \frac{\partial f_{3}}{\partial y}\right](x+Z) } \\
& +\left[(1-m)\left(\frac{\partial f_{3}}{\partial z}-\frac{\partial f_{3}}{\partial y}\right)-m \frac{\partial f_{1}}{\partial y}\right](z+X)
\end{aligned}
$$

where all the partial derivatives are evaluated at $(\tilde{u}, \tilde{v}, \tilde{w})$. The derivation of (5.13) and (5.14) decisively use the identities $\tilde{u}=\tilde{W}, \tilde{v}=\tilde{V}, \tilde{w}=\tilde{U}$. The explicit values of the partial derivatives after some simplification with reference to $(5.9)$ and $(5.10)$ are

$$
\begin{array}{lll}
\frac{\partial f_{1}}{\partial x}=2 \tilde{u}-1+\tilde{v}+\frac{1}{1-2 m} & \frac{\partial f_{3}}{\partial x}=0 \\
\frac{\partial f_{1}}{\partial y}=\frac{\tilde{u}}{1-\tilde{v}} \cdot \frac{1}{1-2 m} & \frac{\partial f_{3}}{\partial y}=\frac{\tilde{w}}{1-\tilde{v}} \cdot \frac{1}{1-2 m} \\
\frac{\partial f_{1}}{\partial z}=0 & \frac{\partial f_{3}}{\partial z}=2 \tilde{w}-1+\tilde{v}+\frac{1}{1-2 m} .
\end{array}
$$

It is not difficult to check that both of the eigenvalues of the system (5.14) are in magnitude less than 1 . The characteristic equation of $(5.13)$ reduces to

$$
\lambda^{2}-\lambda+(\tilde{v} /(1-\tilde{v}))(1-2 \tilde{v})(1-2 m)-\left[2 m /(1-2 m)^{2}\right]=0 .
$$

Direct analysis reveals that the roots of this equation are in magnitude at most 1 , if and only if

$$
\tilde{v} /(1-\tilde{v})(1-2 \tilde{v})>\left[2 m /(1-2 m)^{3}\right]
$$

The largest $m$ consistent with (5.16) and (5.11) is the smallest positive solution $m * *$ of the equation

$\frac{[1-2 \sqrt{m(1-m)}]}{1-2 m}=\frac{3-\frac{2 m}{(1-2 m)^{3}}+\sqrt{1-\frac{12 m}{(1-2 m)^{3}}+\frac{4 m^{2}}{(1-2 m)^{6}}}}{4}$

It can be shown that a positive $m^{* *}$ exists and obeys the inequality $m^{* *}<m^{*}=$ $1 / 10$. It follows that the equilibria (5.12) are locally stable if and only if $m$ satisfies $0<m<m^{* *}$. Notice that the symmetric equilibria persist for a larger range of $m$ up to $m^{*}=1 / 10$ but in the range $m^{* *}<m<m^{*}$ they cease being locally stable.

\section{Discussion}

This study is one of a series of works in which interacting genetic systems are analyzed. The more complex system is obtained from a simpler system by superimposing small interaction and perturbation factors on the subsystems. We concentrate in this paper on several genetic models for natural and sexual selection with the intermigration flow among the subsystems positive but small.

In most studies of migration selection belance it is assumed that in one niche 
one homozygote carries a selective advantage while in a second niche an alternate homozygote is selectively superior (e.g., see Moran (1959), Maynard-Smith (1966)). An application of Principle I of Section 1 demonstrates that two or more populations (or systems) individually subject to disruptive selection pressures would separately fix but when slight gene flow connects the populations then a stable polymorphism with all types persisting can be attained. The cases of total fixation remain also possible equilibria with non-trivial domains of attraction. Thus, for a single niche situation subject to disruptive selection, only fixation is possible. On the other hand, a multiniche (at least two niches) version of the same model with disruptive selection in force in each niche now provides possibilities both of fixation (monomorphism) and polymorphism and which occurs depends on the nature of the initial compositions of the subpopulations or factors other than the forces of natural selection.

In most mathematical modeling of a single genetic system producing a stable polymorphism, it is customarily postulated that there is some clear mechanism operating to the advantage of one of the types and another mechanism favoring an alternative type. For example, in the case of selection balance, polymorphism can be maintained by such factors as heterozygote advantage, selective advantage conferred on different homozygotes in the two sexes, oppositely directed selective pressures in the haploid versus diploid phase, etc. The fact that opposing pressures maintain the equilibrium usually means that if one of these pressures is removed the other force effective alone will bring the population to an obvious fixation. In contrast, our general principle of Section 1 tells us that it is unnecessary to have distinctive balancing forces underlying the existence of stable polymorphism. There merely should be the possibility for a particular type to be retained in some subsystem of the whole system. This situation could transpire owing to the nature of the initial composition of the population, rather than any special pressure favoring the type. For example, in a situation of disruptive selection, the $A A$ homozygote may be selectively inferior to the $B B$ homozygote but if $A A$ is slightly advantageous over $A B$ and the initial population consists abundantly of $A$ genes, then the $A A$ type can be established. Thus, founder effects, sampling and statistical fluctuation, and changing environmental conditions may be important initial factors causing a local population to be mostly of one type. The results could be germane to situations of heterogeneous environments where wild populations are regularly exposed to some measure of disruptive selection for many characters. Such ideas are important in many of the writings of Wright.

It is important to emphasize that the validity of the polymorphisms of Section 3 requires that the migration rates among the distinct systems be very small although some forms of migration selection (e.g., migration to certain niches favoring certain genotypes) may be in force. When migration rates are not small then the various systems (or niches) blend and the ultimate effects of all the selection parameters are complex. On the other hand, when the migration rates are sufficiently small then the selection effects in each niche strongly dominate in their individual niche while the remaining niches help to keep a slight flow of alternative genotypes, thereby maintaining a global stable polymorphism.

Many of the stable polymorphisms established by virtue of the principles of Section 1 for multi niche-selection (natural or sexual) balance have the property that a preponderance of one homozygote is maintained in one niche while a preponderance of an alternative homozygote exists in a second niche and so on for the other niches. It is reasonable to speculate that forms of habitat selection confer an advantage, say on $B B$ in niche $2, A A$ is favored in niche 1 while the heterozygote (or hybrid type) bears marked disadvantage to both homozygotes in each of the niches and a global balance may be preserved.

Consider also the situation of a haploid two locus population. Feldman [1971] indicates that in any haploid two locus population practicing random mating, selection pressures alone cannot produce a stable polymorphism. Thus, if polymorphism obtains, some other influence apart from selection pressures should be operating. Along these lines, it is implicit in the work of Raper and others that random mating is not applicable to a number of haploid models of fungi populations e.g., Schizophyllum Commune and indeed, certain incompatibility mechanisms are in force. For such a two locus haploid population, Feldman showed that a stable polymorphism can be obtained attributable to the incompatibility mating pattern alone.

In Section 4 we show under wide conditions on the selection parameters that a polymorphism is possible for the multi niche version of the same haploid two locus population.

Natural populations live in heterogeneous environments and must therefore be exposed to disruptive and/or directional selection pressure for many characters. If our findings of Sections 2-5 are extrapolated to multi niche situations, there would emerge patterns of clusters of different homozygotes suitably interspersed geographically.

The existence of sharp clines and/or mosaics of different types can also be explained along the lines of the examples of Sections 2-5 involving arbitrary levels of selection pressures (which may or may not differ over two neighboring habitats) provided slight gene flow among neighboring habitats occurs. Other attempts to understand the evolution of morph-ratio clines usually stress rapid changes in selective value as a function of habitat (e.g., see Clark (1966)). Our analysis shows that this assumption of strong selection is unnecessary to assure global polymorphism or sharp clines although it could be a contributing factor (sometimes the main one) in certain cases.

It is important to realize that the nature of local stability for the pure equilibria, for example algebraic versus geometric local convergence in the separate niches is irrelevant to the main conclusion of Principle I. 
The examples of Sections 3 and 4 for the incompatibility model of Section 5 and the multi niche assortative mating model of Karlin and McGregor [1972], the rate of fixation of the pure equilibria is algebraic. Generally, forms of sexual selection lead to algebraic rates of fixation while effects of natural selection bring geometric convergence. Our general principle applies in both situations. However, there is a difference of order of magnitude allowed for the migration parameters in the two cases yielding polymorphisms. When algebraic as against geometric convergence is operating in the corresponding one niche model at a specified equilibrium, it manifestly takes longer to fix in such a population. In this circumstance, it can be proved that the order of migration assuring stable polymorphism is the square root of that where geometric convergence is operating. Therefore more possibilities of polymorphism arise with algebraic convergence (in situations of sexual selection) than in the case of geometric convergence (natural selection). In particular, migration rates can be larger in the cases of assortative mating producing polymorphism than in the cases where disruptive selection is effective.

An interesting consequence of Principle II concerns the problem of the minimum number of niches required to guarantee the existence of $p$-types in a stable polymorphic state (see the close of Section 3). Assuming sexual or natural selection operating in each niche fixes a different type, then a minimum of $p$ niches with slight migration flow between them can maintain a global stable polymorphism with all $p$ types represented (see Section 1).

When it is possible to have more than one type kept in a stable state in some or several niches then the minimum required number of niches to maintain all $p$ types is diminished from $p$. There is some similarity of this result and the Gause exclusion principle of ecology.

The principles of Section 1 have, perhaps, two striking general qualitative implications concordant with much biological phenomena:

(a) Systems with simple monomorphic equilibria when combined with slight interactions (gene flow) among them produce a complex system with stable polymorphic equilibria.

(b) Complex systems when combined with slight migration between them produce even more complex systems with more possibilities and representations of stable polymorphisms.

\section{REFERENCES}

Clarke, B. C. 1966. The evolution of morph-ratio clines, Amer. Natur. 100, 389-402. Bodmer, W. F. ANd Parsons, P. A. 1962. Linkage and recombination in evolution, Advan. Genet. 11, 1-100.

Feldian, M. 1971. Equilibrium studies of two locus haploid populations with recombination, Theor. Pop. Bio. 2, 299-318.
Karlin, S. AND Feldman, M. W. 1968. Further analysis of negative assortative mating, Genetics 59, 117-136.

Karlin, S. AND MCGregor, J. 1972. Polymorphisms for genetic and ecological systems with weak coupling, Theor. Pop. Bio. 3.

KarLIN, S. AND McGregor, J. 1973. Polymorphisms for multi-locus selection system with tight linkage, unpublished.

LEVENE, H. 1953. Genetic equilibrium when more than one ecologieal niche is available, Amer. Natur. 87, 311-313.

Maynard Smith, J. 1966. Sympatric speciation, Amer. Natur. 100, 637-650.

Moran, P. A. P. 1959. The theory of some genetical effects of population subdivision, Aust. J. Biol. Sci. 12, 109-116.

Moran, P. A. P. 1962. "The Statistical Processes of Evolutionary Theory," The Clarendon Press, Oxford.

Parsons, P. A. 1963. Migration as a factor in natural selection, Genetica 33, 184-206.

Prout, T. 1968. Sufficient conditions for multiple niche polymorphism, Amer. Natur. 102, 493-496.

SPIETH, P. T. 1971. A necessary condition of equilibrium in systems exhibiting selfincompatible mating, Theor. Pop. Bio. 2, 404-418.

WorkMAN, P. L. 1964. The maintencance of heterozygosity by partial negative assortative mating, Genetics 50, 1369-1382. 